\title{
Conformal decision-tree approach to instance transfer
}

\author{
S. Zhou ${ }^{1}$ - E. N. Smirnov ${ }^{1}$ - G. Schoenmakers ${ }^{1}$. \\ R. Peeters ${ }^{1}$
}

Published online: 17 June 2017

(C) The Author(s) 2017. This article is an open access publication

\begin{abstract}
Instance transfer for classification aims at boosting generalization performance of classification models for a target domain by exploiting data from a relevant source domain. Most of the instance-transfer approaches assume that the source data is relevant to the target data for the complete set of features used to represent the data. This assumption fails if the target data and source data are relevant only for strict subsets of the input features which we call "partially input-feature relevant". In this case these approaches may result in suboptimal classification models or even in a negative transfer. This paper proposes a new decision-tree approach to instance transfer when the source data are partially input-feature relevant to the target data. The approach selects input features for tree nodes using univariate transfer of source instances. The instance transfer is guided by a conformal test for source relevance estimation. Experimental results on real-world data sets demonstrate that the new decision-tree approach is capable of outperforming existing instance-transfer approaches, especially, when the source data are partially input-feature relevant to the target data.
\end{abstract}

Keywords Instance transfer · Classification · Decision trees · Conformal prediction framework

Mathematics Subject Classification (2010) 97R40

\section{S. Zhou}

shuang.zhou@maastrichtuniversity.nl

E. N. Smirnov

smirnov@maastrichtuniversity.nl

G. Schoenmakers

gm.schoenmakers@maastrichtuniversity.nl

R. Peeters

ralf.peeters@maastrichtuniversity.nl

1 Department of Data Science and Knowledge Engineering, Maastricht University, P.O.BOX 616, Maastricht, 6200 MD, The Netherlands 


\section{Introduction}

Instance transfer for classification has received significant attention in the last two decades [15]. It assumes the presence of two domains: a target domain (the domain of interest) and a source domain (the auxiliary domain). Both domains are represented by the same set of input features and share the same class labels but differ in the underlying distributions. The goal of instance transfer is to improve classification models in the target domain by utilizing data from the source domain.

The effectiveness of instance transfer depends on the relevance of the source domain to the target domain [19]. Since the distributions of the domains are usually unknown, the relevance can be estimated by the relevance of the source data to the target data. Existing instance-transfer approaches estimate the relevance of source data to the target data in a multivariate setting, i.e. they consider all the input features when estimating the relevance (e.g. [7, 9]). If the relevance of source data is inadequate, these approaches either stop instance transfer, or select and transfer only those source instances that are estimated to be relevant (if any). Although this strategy seems reasonable, it may still fail when the source data are relevant to the target data for strict subsets of the input features. In this situation the effectiveness of instance transfer depends on the proportion of input features for which the target data and source data are relevant. More precisely,

- if the source data are relevant to the target data w.r.t. the majority of input features, transferring the selected source instances can improve the generalization performance of the final classification model; i.e., we have positive instance transfer. However, we note that although the instance transfer is positive, it may be sub-optimal. This is because the final model still contains information of source instances provided by irrelevant features.

- item if the source data are irrelevant to the target data w.r.t. the majority of input features, transferring the selected source instances can degrade the generalization performance of the final classification model; i.e., we have negative instance transfer.

This paper addresses the problem of sub-optimal positive instance transfer and the problem of negative instance transfer in the context of partial input-feature relevance. For this purpose a new decision-tree approach is proposed. The approach transfers parts of the source data that are similarly distributed as the target data for strict subsets of the input features. It employs the standard decision-tree algorithm [16] to construct trees. Univariate instance transfer is performed on the level of feature selection for test nodes of decision trees. More precisely, at each test node the approach first selects for every feature the largest source subset which is relevant to the target data when only considering this feature. The relevance of source instances is decided by a statistical test, namely conformal test [22]. Then, the approach estimates the class relevance of this feature on the target data and the selected source subset using some measures (e.g., Information Gain, Gain Ratio, etc. [16]). Once the class relevance of all features have been estimated, the approach selects the feature with the highest class relevance for this test node. We note that constructing a decision tree consists of a series of such steps of univariate instance transfer and feature selection. Thus, our decision-tree approach is essentially an embedded multi-variate feature selection approach to instance transfer based on univariate instance transfer and feature selection.

The key feature of our approach is that by using univariate instance transfer it selects different largest source subsets for different input features, and the selection error is controlled through the conformal test. In this context, we note that for every test node: 
- $\quad$ if the selected source subsets are empty for all the features, the feature selection is not affected by the univariate instance transfer. In this case it selects a feature that can best predict class labels on the target data (sub-)set associated with this node. The source data are irrelevant to the target data (sub-)set when considering that feature.

- if the selected source subsets are not empty, the feature selection is affected by the univariate instance transfer. In this case it selects a feature that can best predict class labels on the associated target data (sub-)set and selected source subset. The selected source data are statistically relevant to the target data (sub-)set when considering that feature.

Therefore, our approach selects features for test nodes either based on target data, or based on target data and statistically relevant source instances. This avoids negative transfer and sub-optimal positive transfer.

The remainder of this paper is structured as follows. Section 2 provides an overview of related work. The task of instance transfer is formalized in Section 3. Section 4 presents the conformal test employed. Our decision-tree approaches to instance-transfer are introduced in Section 5. Experiments are given in Section 6. Finally, Section 7 concludes the paper and discusses possible directions of future work.

\section{Related work}

Existing instance transfer approaches can generally be divided into two categories: feature selection based approaches and source-subset selection based approaches. Feature selection based approaches first find a low-dimensional feature space where the target data and source data are relevant. Then, classification models are trained on the combination of target data and source data in the low-dimensional space. One well-known approach belonging to this family is the Maximum Mean Discrepancy Embedding (MMDE) [13]. MMDE first learns a kernel matrix corresponding to a nonlinear transformation that projects the target data and source data to a latent space, in which the distance between the two data sets is minimized. The distance between the data sets is measured by Maximum Mean Discrepancy (MMD) [4]. Then, MMDE applies Principal Component Analysis (PCA) [8] on the learned kernel matrix to obtain a low-dimensional representation for the target data and source data jointly. Finally, any traditional machine learning algorithm can be applied to learn a model on the combination of target and source data in the low-dimensional feature space. MMDE is effective yet not computationally efficient. To address this issue, the same authors have proposed another approach in [14], namely Transfer Component Analysis (TCA), which is as effective as MMDE but more efficient in terms of computing the nonlinear transformation.

Another feature selection based approach is Feature selection by Maximum Mean Discrepancy (f-MMD) [20]. Rather than finding a low-dimensional representation for the target data and source data jointly, f-MMD identifies a subset of features (called variant features) which contribute the most to the MMD score and excludes them from the original feature space. The problem of finding variant features is formulated as a convex optimization problem. More precisely, a weight matrix, the diagonal of which corresponds to the weights of all the features, is incorporated in the MMD calculation. The variant features are expected to receive higher weights after optimization since they minimize the negative MMD score in the objective function. That is to say the variant features are defined as those that contribute most to maximizing the MMD between data sets. 
Analyzing these feature selection based approaches, we note that there are mainly two drawbacks. First, they may impair geometric or statistical properties of the original data due to the dimensionality reduction. Second, these approaches learn the low-dimensional space in an unsupervised manner and dismiss the relevance of features to class labels. Some of the removed features may have strong class relevance and influence the performance of resulting models.

Source-subset selection based approaches to instance transfer first select a subset of source instances that are relevant to the target data, and then train classification models on the selected source subset in addition to the target data. Source-subset selection can be done in two ways: soft selection and hard selection. The soft selection picks the source instances implicitly. It assigns weights to source instances proportionally to their relevance to the target data. Thus, less (more) relevant source instances have less (more) influence on the final classification model. The hard instance selection picks the source instances explicitly. It directly selects source instances that influence the final classification model in addition to the target data.

Soft selection has been implemented in several boosting-based approaches, e.g., TrAdaBoost [7] and Dynamic-TrAdaBoost [2]. These approaches are similar to the AdaBoost algorithm [17] but employ two opposite weight-update schemes depending on the type of the instances: (1) the weights of misclassified target instances are being increased, and (2) the weights of misclassified source instances are being decreased. In theory, assuming that the number of iterations approaches infinity, the average weighted training loss of boostingbased algorithms on the source data will converge to 0 [7]. This implies that in this case relevant source instances will be classified correctly and irrelevant source instances will receive a weight of 0 ; i.e., there will be a perfect selection of the source instances. However, in practice when most of the source instances are irrelevant, these algorithms are likely to stop at the first few iterations because the training error on target data exceeds 0.5 in early iterations. In this case, irrelevant source instances are not filtered out and pose negative effects on the final model.

Hard selection is implemented in several bagging-based approaches. There are two types of implementation: direct and indirect. Double-Bootstrap [12] is an example of direct implementation. It first constructs an ensemble of classification models trained on bootstrap samples from the target data. Then the ensemble classifies the source instances and those of them that are correctly classified are selected. Thus, when most of the source instances are irrelevant, this approach tends not to select source instances; i.e., instance transfer stops.

TraBagg [9] is an example of indirect implementation of the hard instance selection. It first randomly generates a set of bootstrap samples from the combined target and source data, and then trains several base classification models on those samples. Finally, a subset of the base classification models are selected by minimizing the empirical error on the target data. This means that source subsets that are contained in the bootstrap samples are indirectly selected through selecting the base models. When the relevance of the source data to the target data is low, TraBagg requires a large number of bootstrap iterations to filter out irrelevant source instances which makes it computationally inefficient.

Unlike feature selection based approaches, source-subset selection based approaches do not suffer from any information loss. However, they assume the existence of a subset (or some subsets) of the source data which is (are) likely to be drawn from the target distribution. Such a source subset may not exist when the source data are relevant to the target data for strict subsets of the input features. In this case, these approaches either disregard the source data or select an inadequate source subset which may lead to sub-optimal or even negative instance transfer. 
In the next sections we consider how to avoid sub-optimal transfer and negative instance transfer when the source data are partially input-feature relevant to the target data. For that purpose, we develop a decision-tree approach to instance transfer. Our approach is essentially a source-subset selection based approach. ${ }^{1}$ It differentiates itself from other existing source-subset selection approaches by employing univariate instance transfer that selects different relevant source subsets for different input features.

\section{Task formalization}

Let $X$ be a feature space with $I$ features $X^{i}, i \in\{1,2, \ldots, I\}$, and $Y$ be a (finite) class set. A domain is defined as a tuple consisting of a labeled space $(X \times Y)$ and a probability distribution $P$ over $(X \times Y)$. We consider first a domain $\left\langle(X \times Y), P_{T}\right\rangle$ that we call a target domain. The target data set $T$ is a multi set of $m_{T}$ instances $\left(x_{t}, y_{t}\right) \in(X \times Y)$ drawn from the target distribution $P_{T}$ under the i.i.d. assumption. Given a test instance $x_{m_{T}+1} \in X$, the target classification task is to find an estimate $\hat{y} \in Y$ for the true class of $x_{m_{T}+1}$ in accordance with $P_{T}$.

Let us consider a second domain $\left\langle(X \times Y), P_{S}\right\rangle$ that we call a source domain. The source data $S$ is a multi set of $m_{S}$ instances $\left(x_{s}, y_{s}\right) \in X \times Y$ drawn from the source distribution $P_{S}$ under the i.i.d. assumption. Assuming that the source domain is relevant to the target domain, we define the instance-transfer classification task as a task to find an estimate $\hat{y} \in Y$ for the true class of $x_{m_{T}+1}$ in accordance with $P_{T}$ using source data $S$ as auxiliary training data.

\section{Conformal test to decide on source relevance}

In our decision-tree approaches to instance transfer, the conformal test (CT) [22] is employed to estimate the relevance of the source data to the target data. In the following, we first describe the CT and the $p$-value function it employs. Then, we provide an approximation of the $p$-value function that is computationally efficient. Finally, we explain and compare two different ways to use the $\mathrm{CT}$ for source relevance estimation.

\subsection{Conformal test}

The CT is proposed under the exchangeability assumption of data generation [3]. ${ }^{2}$ It works with data sequences. Given a target data sequence $T$ and a source data sequence $S$, it decides the relevance of $S$ to $T$ by testing the null hypothesis that the concatenated data sequence $T S$ has been generated from the target distribution $P_{T}$ under the exchangeability assumption.

To test the null hypothesis, the CT makes use of the conformal prediction framework that was introduced in $[18,21]$. The test employs the nonconformity scores of subsequences of $T S$ as test statistics for the null hypothesis. The nonconformity score of a subsequence

\footnotetext{
${ }^{1}$ Although our approach includes feature selection, it selects features only based on class relevance which differentiates itself from the feature selection based approaches for instance transfer.

${ }^{2}$ The exchangeability assumption is a weaker assumption than the i.i.d. assumption. A finite sequence of random variables is exchangeable if the joint distribution of these variables is invariant under any permutation of the indices.
} 
can be computed based on the nonconformity scores of the instances contained in the subsequence. Given the concatenated data sequence $T S$, the nonconformity score $\alpha_{(x, y)}$ of an instance $(x, y) \in T S$ is a positive real number that indicates how strange the instance $(x, y)$ is w.r.t. the sequence $T$. To compute the instance nonconformity scores we need an instance nonconformity function $A$. If $(X \times Y)^{(*)}$ represents the set of all sequences over $(X \times Y)$, the instance nonconformity function $A$ is a mapping from $(X \times Y)^{(*)} \times(X \times Y)$ to $\mathbb{R}^{+} \cup\{+\infty\}$ that measures the degree of strangeness of an instance in relation to a sequence.

To compute the sequence nonconformity scores we need a sequence nonconformity function. Given the combined sequence $T S$, a subsequence $U$ of some elements of $T \cup S$, the sequence nonconformity function returns a score $\alpha_{U}$ indicating how strange the subsequence $U$ is with respect to all subsequences with size $|U|$ of the data sequence $T S$.

Definition 1 (Sum Sequence Nonconformity Function) Given an instance nonconformity function $A$, data sequences $T$ and $S$, and a subsequence $U$ of some elements of $T \cup S$, the sum sequence nonconformity function $A^{*}:(X \times Y)^{(*)} \times(X \times Y)^{(*)} \rightarrow \mathbb{R}^{+} \cup\{+\infty\}$ is defined as

$$
A^{*}(T, U)=\sum_{(x, y) \in U} \alpha_{(x, y)},
$$

where $\alpha_{(x, y)}=\left\{\begin{array}{lr}A(T \backslash\{(x, y)\},(x, y)), & \text { if }(x, y) \in T \\ A(T,(x, y)), & \text { otherwise. }\end{array}\right.$

The CT employs sequence nonconformity scores as test statistics. The $p$-value function of the CT is defined as follows.

Definition 2 ( $p$-Value Function) The $p$-value function is a function $t:(X \times Y)^{(*)} \times(X \times$ $Y)^{(*)} \rightarrow[0,1]$ defined as:

$$
t(T, S)=\frac{\left|\left\{U \in \mathcal{P}\left(T S, m_{S}\right): \alpha_{U} \geq \alpha_{S}\right\}\right|}{\left|\mathcal{P}\left(T S, m_{S}\right)\right|},
$$

where $\mathcal{P}\left(T S, m_{S}\right)$ is the set of all subsequences of $T S$ with length $|S|=m_{S}, \alpha_{U}$ and $\alpha_{S}$ are sequence nonconformity scores returned by $A^{*}(T, U)$ and $A^{*}(T, S)$, respectively.

The validity of the $p$-value function $t$ has been proven in [22]. The $p$-value returned by the function $t$ indicates the likelihood that the sequence $T S$ has been generated by the target distribution $P_{T}$ under the exchangeability assumption. The higher the $p$-value is, the more relevant the source sequence is to the target sequence. Therefore, this $p$-value can be viewed as an asymmetric measure of relevance of the source data to the target data.

The CT employs the $p$-value function $t$ for testing the exchangeability of the combined data sequence $T S$. The source data sequence is relevant to the target data sequence at a significance level $\epsilon_{t} \in[0,1]$ if and only if the returned $p$-value is greater than or equal to $\epsilon_{t}$

\subsection{Approximation of the $p$-value function}

Notice that the sum sequence nonconformity function $A^{*}(T, U)$ is independent of the ordering of the sequence $U$, so that the nonconformity score of a set is equal to the nonconformity score of any sequence consisting of all elements of that set. We can use the set nonconformity score to define the $p$-value function $t$ for data sets, and this equivalent definition of $t$ is used in the rest of the paper. 
Given the target data set $T$ and source data set $S$, let $\mathcal{C}\left(T \cup S, m_{S}\right)$ be the set consisting of all subsets of $T \cup S$ with size $m_{S}$. For any of these subsets, there are $m_{S}$ ! orderings of elements in that set. Therefore, the cardinality of $\mathcal{P}\left(T S, m_{S}\right)$ is equal to $\left|\mathcal{C}\left(T \cup S, m_{S}\right)\right| \times$ $m_{S}$ !. We re-write the $p$-value function $t$ as follows:

$$
\begin{aligned}
t(T, S) & =\frac{\left|\left\{U \in \mathcal{P}\left(T S, m_{S}\right): \alpha_{U} \geq \alpha_{S}\right\}\right|}{\left|\mathcal{P}\left(T S, m_{S}\right)\right|} \\
& =\frac{\left|\left\{U \in \mathcal{P}\left(T S, m_{S}\right): \alpha_{U} \geq \alpha_{S}\right\}\right| / m_{S} !}{\left|\mathcal{P}\left(T S, m_{S}\right)\right| / m_{S} !} \\
& =\frac{\left|\left\{U \in \mathcal{C}\left(T \cup S, m_{S}\right): \alpha_{U} \geq \alpha_{S}\right\}\right|}{\left|\mathcal{C}\left(T \cup S, m_{S}\right)\right|}
\end{aligned}
$$

Now, the function $t$ is analogous to the $p$-value function in the Wilcoxon rank-sum test (see, e.g., [10], Chapter 1). Hence, we approximate the set $p$-value through the Wilcoxon rank-sum test for big data sets for which enumerating all combinations in $\mathcal{C}\left(T \cup S, m_{S}\right)$ is intractable. More precisely, we assign ranks from 1 to $m_{T}+m_{S}$ to instances in $T \cup S$ arranged in ascending order of nonconformity scores. The nonconformity score $\alpha_{U}$ of any subset $U$ of $T \cup S$ with size $m_{S}$ is then replaced by the rank-sum $W$ that equals to $\sum_{(x, y) \in U} R_{(x, y)}$, where $R_{(x, y)}$ is the rank of the nonconformity score of instance $(x, y) \in U$. Accordingly, $\alpha_{S}$ is replaced by the sum of ranks of all instances in $S$ denoted by $W_{S}$. In this way the probability $P\left(W \geq W_{S}\right)$ that the rank-sum of any $m_{S}$ instances is bigger than that of the source instances is approximately equal to $t(T, S)$.

When $|T \cup S|>30$, according to the law of large numbers, the rank-sum $W$ approximately follows a normal distribution with expectation $\mu$ equal to $\frac{1}{2} m_{S}\left(m_{T}+m_{S}+1\right)$, and variance $\sigma^{2}$ equal to $\frac{1}{12} m_{T} m_{S}\left(m_{T}+m_{S}+1\right)$ [10]. In this case, $P\left(W \geq W_{S}\right)$ and, thus, the $p$-value $t\left(T S, m_{S}\right)$, can be easily approximated from this normal distribution .

\subsection{Estimating individual relevance and set relevance using the $p$-value function}

As mentioned in Section 4.1, the $p$-value returned by the function $t$ can be viewed as an asymmetric measure of relevance of the source data to the target data. Since the $p$-value function $t$ can be applied to source data with arbitrary size, it allows for measuring the relevance of the source data in two different ways. When the size of the source data equals $1\left(m_{S}=1\right)$, function $t$ estimates the individual relevance of a source instance $\left(x_{s}, y_{s}\right)$ with value $t\left(T,\left\{\left(x_{s}, y_{s}\right)\right\}\right)$. When the size of the source data is greater than $1\left(m_{S}>1\right)$, function $t$ estimates the relevance of the source set as a whole with value $t(T, S)$. An elaborate analysis of properties of individual relevance and set relevance estimated using function $t$ can be found in [23].

Comparing to individual relevance, set relevance usually is a more precise instrument for estimation of source relevance. For (1), we have that if $S=\left\{\left(x_{s}, y_{s}\right)\right\}$ then $m_{S}=1$, and $|C(T \cup S, 1)|=m_{T}+1$, whereas the numerator expression is a positive integer. Consequently, the number of possible individual $p$-values is bounded by $m_{T}+1$. If $m_{S}>1$, the number of possible set $p$-values is bounded by $\left|\mathcal{C}\left(T \cup S, m_{S}\right)\right|$, which quickly grows much larger than $m_{T}+1$. Therefore, the set $p$-values can better distinguish sets with different nonconformity scores.

Source-subset selection based on individual relevance is computationally more efficient than that based on set relevance. Assume that all instances in the source data $S$ are sorted by increasing order of nonconformity scores. According to Definition 2, we have that the 
individual relevance of the source instance with index $s(s>1)$ is always less than or equal to that of the source instance with index $s-1$, i.e., $t\left(T,\left\{\left(x_{s}, y_{s}\right)\right\}\right) \leq t\left(T,\left\{\left(x_{s-1}, y_{s-1}\right)\right\}\right)$. That is to say the individual relevance is a decreasing function of the index $s$, and through the index $s$, it is also a decreasing function of the nonconformity score. When individual relevance is employed to select the largest subset of source instances that passes the CT at a certain significance level $\epsilon_{t}$, we can simply apply binary search on the sorted source set to quickly find the last instance that has a $p$-value no less than $\epsilon_{t}$. The largest source subset is then formed by adding all the instances before this instance and the instance itself.

The set relevance in general is not a monotonic function of the index $s$, and is not a monotonic function of the nonconformity score. Let $S_{S}$ be the subset of $S$ consisting of the first $s$ instances of the sorted data $S$. For each $s$ we may have either $t\left(T, S_{S}\right) \leq t\left(T, S_{s-1}\right)$ or $t\left(T, S_{S}\right) \geq t\left(T, S_{s-1}\right)$. This result is not so straightforward, and to provide an intuition we illustrate that with the following example. Assume that a set $T \cup S$ consists of target instances $t_{1}, t_{2}, t_{3}$ associated with nonconformity scores $1,4,5$, and source instances $s_{1}, s_{2}$, $s_{3}$ associated with nonconformity scores 2,3,6 (note that the source instances are sorted by increasing order of the nonconformity scores). In this case, we have $t\left(T, S_{1}\right)=0.75$, $t\left(T, S_{2}\right)=0.8$ and $t\left(T, S_{3}\right)=0.5$. Due to the non-monotonicity, source-subset selection based on set relevance is computationally inefficient. It may involve repeated application of the $p$-value function $t$.

Although set relevance in general is not a monotonic function of nonconformity score, it decreases when sequentially adding source instances from the sorted source data whose nonconformity scores are greater than or equal to the largest nonconformity score $\alpha_{T_{\max }}$ in the target data [23]. Let $m$ be the index of the first source instance in the sorted data $S$ that has a nonconformity score greater than or equal to $\alpha_{T_{\max }}$. Set relevance is a decreasing function of the index $s$ in the interval $\left[m, m_{S}\right]$, see Theorem 1 in [23].

\section{Decision-tree approaches to instance transfer}

In this section, we introduce our instance-transfer decision trees. We first present the standard algorithm for the induction of decision trees. Then, we describe the algorithms for building instance-transfer decision trees in detail.

\subsection{Decision trees}

Decision trees are among the most popular models in machine learning [16]. A decision tree can be defined recursively as a leaf node assigning a class or a test node with a decision tree for each test outcome. A decision tree is induced in a top-down manner from a target data set $T$ by calling a single recursive method BUILD TREE given in Algorithm 1. The method employs three functions given below:

- Terminate $(T)$ is a boolean function that returns true if the data $T$ satisfies a stopping criterion; otherwise, it returns false.

- ClassRelevance $\left(T, X^{i}\right)$ is a real-valued function that returns an estimation of the class relevance of the feature $X^{i}$ using the data $T$.

- $\operatorname{Split}\left(T, X^{i}\right)$ is a function that splits the data $T$ into subsets $T_{j}$ according to the values of the feature $X^{i}$. It returns a set $T_{s p l i t}$ of subsets $T_{j}$.

The decision tree induction starts with the function BUILDTREE applied on the entire data set $T$. The function first tests whether the data $T$ satisfies a stopping criterion (e.g., 
entropy $=0$ ). If so, it creates a leaf node and stores the number $s_{y}$ of training instances in $T$ for each class $y \in Y$ that arrive at this node. If the training data does not satisfy the stopping criterion, the function creates a node $N$. To find a feature to test for $N$, the class relevance of all the features $X^{i}$ is estimated. Some commonly used measures for estimating class relevance are Information Gain, Gain Ratio, etc., see [16]. Once the class relevances of all the features have been obtained, the feature with the highest estimate is selected for the node $N$ with a corresponding test. The data $T$ is then split into subsets $T_{j}$ and the function BUILDTREE is recursively called for each $T_{j}$.

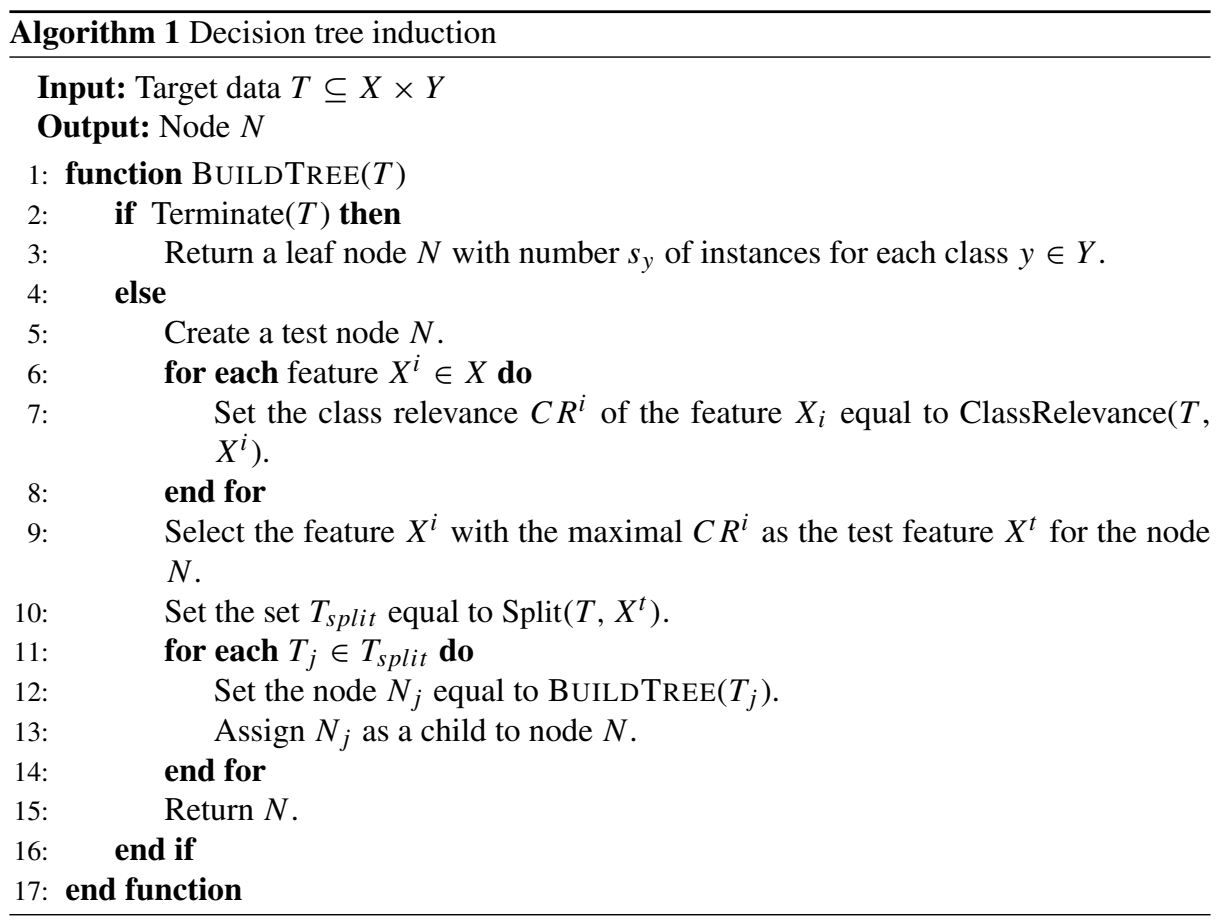

\subsection{Instance transfer decision trees}

There are two approaches to implement instance transfer with decision trees using the conformal test CT. Below we sketch both of them.

- Decision trees with pre-training source-subset selection. This approach selects the relevant source subset prior to inductive decision-tree construction. More precisely, given a significance level $\epsilon_{t}$ the approach first selects the largest source subset $S_{R} \subseteq S$ that can pass the CT at $\epsilon_{t}$. Then, the subset $S_{R}$ is added to the target data and a decision tree is trained on the union $T \cup S_{R}$. Since the relevance of the source data can be estimated in two ways, we introduce:

- Decision trees with pre-training selection based on individual relevance, denoted as PreIR-DT;

- Decision trees with pre-training selection based on set relevance, denoted as PreSR-DT. 
- Decision trees with embedded source-subset selection. This approach selects the largest source subset that passes the CT independently for each feature $X^{i},(i=$ $1,2, \ldots, I)$. The class relevance of $X^{i}$ is estimated on the union of the target data and the selected subset. The feature with the highest class relevance is selected. This implies that univariate instance transfer and feature selection are both embedded in the decision-tree induction. Since the relevance of the source data can be estimated in two ways, we introduce:

- Decision trees with embedded selection based on individual relevance, denoted as EmbedIR-DT;

- Decision trees with embedded selection based on set relevance, denoted as EmbedSR-DT.

All the four types of decision trees are applicable when the target data and the source data are relevant with respect to all input features. However, when the source data are partially input-feature relevant to the target data, the decision trees with pre-training selection may either transfer sub-optimal source instances, or discard the source instances completely. By contrast, the decision trees with embedded selection can transfer source instances for relevant features. Therefore, we advocate decision trees with embedded selection and detail them in the following section.

\subsection{Induction of decision trees with embedded source-subset selection}

EmbedIR-DT and EmbedSR-DT induce decision trees using the standard decision tree algorithm (shown in Algorithm 1). Instance transfer is implemented in the step of class relevance estimation (step 7). To make this explicit, we have set up a new function TrIRClassRelevance $\left(T, X^{i}\right)$ for EmbedIR-DT, and a new function $\operatorname{TrSR}-C l a s s R e l e v a n c e\left(T, X^{i}\right)$ for EmbedSR-DT to replace the function ClassRelevance $\left(T, X^{i}\right)$ in Algorithm 1.

The function TrIR-ClassRelevance is given in Algorithm 2. It starts with checking if there are sufficient target instances per class at node $N$ for instance transfer. If there are sufficient target instances (i.e., the number of target instances for each class is greater than or equal to a user-defined threshold value $\lambda$ ), the largest source subset that is relevant to the target data $T$ is selected for the feature $X^{i}$. The source relevance is estimated as individual relevance computed by the $p$-value function $t$. To be more specific, the function first builds a projection $T^{i}$ for the target data and a projection $S^{i}$ for the source data in a bivariate space $\left(X^{i} \times Y\right)$. The nonconformity score $\alpha_{t}^{i}$ for each target instance $\left(x_{t}^{i}, y_{t}\right) \in T^{i}$ is calculated w.r.t. $T^{i} \backslash\left\{\left(x_{t}^{i}, y_{t}\right)\right\}$, and the nonconformity score $\alpha_{s}^{i}$ for each source instance $\left(x_{s}^{i}, y_{s}\right) \in S^{i}$ is calculated w.r.t. $T^{i}$. Then, the function sorts the source instances increasingly by nonconformity scores. Since $t\left(T^{i},\left\{\left(x_{s}^{i}, y_{s}\right)\right\}\right)$ is a decreasing function of the index $s$, the individual relevance of source instances are arranged in decreasing order of magnitude. If the individual relevance $p_{m_{S}}^{i}$ of the last instance in the sorted (sub-)set $S^{i}$ is greater than or equal to $\epsilon_{t}$, then the whole $S^{i}$ is transferred. Otherwise, the function proceeds with selecting the largest subset of $S^{i}$ that consists of all instances with individual relevance no less than a given significance level $\epsilon_{t}$. This can be implemented with a simple binary search on the sorted $S^{i}$ to find the last source instance that has individual relevance greater than or equal to $\epsilon_{t}$. The source instances from $S^{i}$ with indices smaller than or equal to $m$ constitute the largest relevant subset $S_{R}^{i}$. The class relevance of $X^{i}$ is then estimated on $T^{i} \cup S_{R}^{i}$ using the class relevance measure of choice (e.g., Information Gain). 


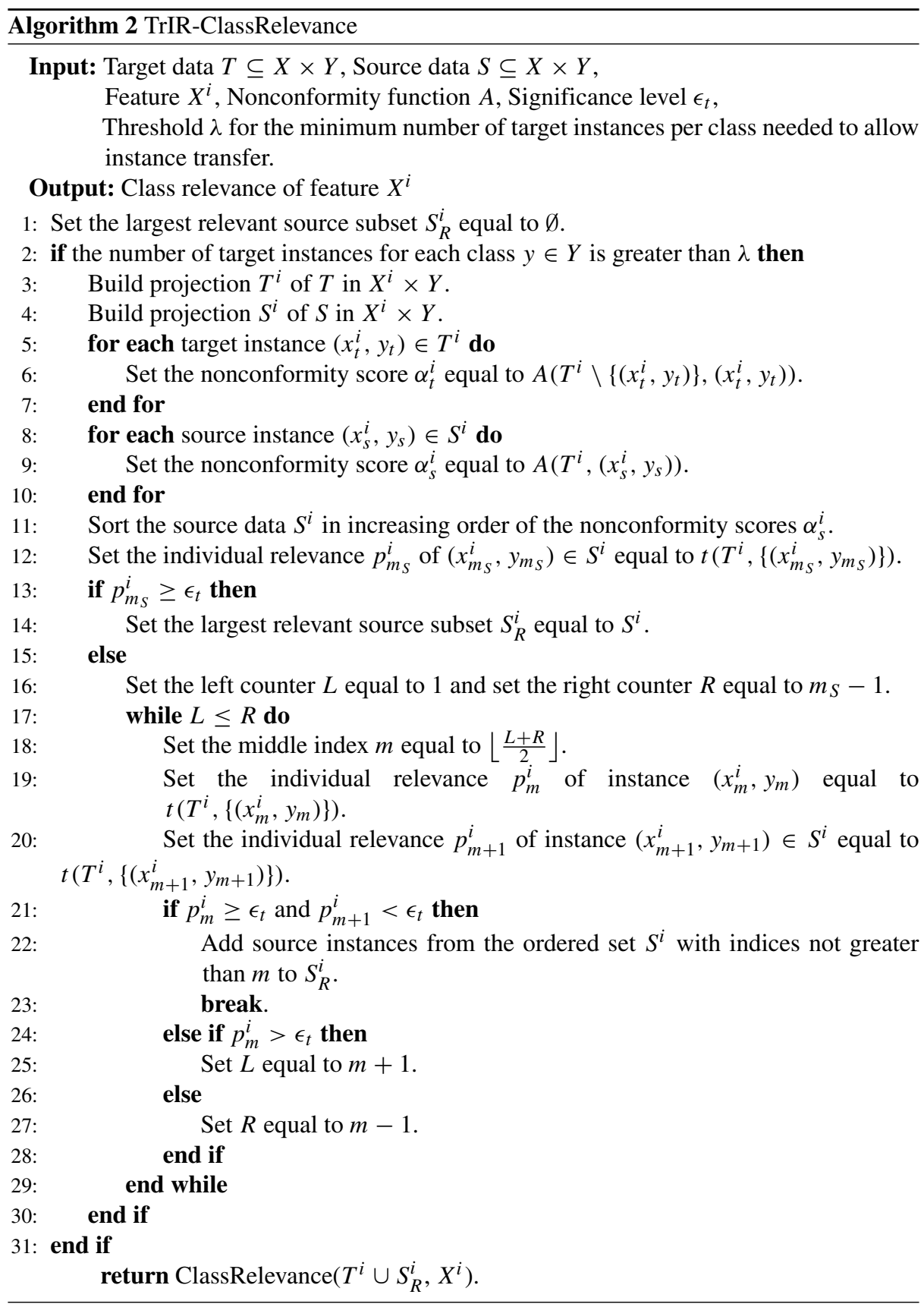

In case there are insufficient target instances available, instance transfer will not be performed and the class relevance of the feature $X^{i}$ is estimated only on the target data. This is meant as a safe-guard against inappropriate instance transfer, which could otherwise result for inaccurate estimates of class relevance. 
The TrSR-ClassRelevance function is given in Algorithm 3. It works in the same way as the TrIR-ClassRelevance function for nonconformity-score computation, but differs in the way of selecting the largest source subset that passes the CT. More precisely, once the nonconformity scores of all target and source instances have been computed, the function first determines the largest nonconformity score $a_{T_{\max }}^{i}$ among all the target instances. Then $S_{R}^{i}$ is initialized by including all the source instances $\left(x_{s}^{i}, y_{s}\right) \in S^{i}$ with nonconformity scores $\alpha_{s}^{i}$ smaller than $\alpha_{T_{\max }}^{i}$. As explained in Section 4.3, the set relevance of $S_{R}^{i}$ decreases as sequentially adding instances from the sorted $S^{i}$ whose nonconformity scores equal to or greater than $\alpha_{T_{\max }}^{i}$. Therefore, if $t\left(T^{i}, S_{R}^{i}\right)$ is bigger than a given significance level $\epsilon_{t}$, binary search can be applied to find the largest superset of $S_{R}^{i}$ that has set relevance greater than or equal to $\epsilon_{t}$. If $t\left(T^{i}, S_{R}^{i}\right)$ is smaller than $\epsilon_{t}$, then the algorithm minimally reduces $S_{R}^{i}$. It sequentially removes instances from the sorted $S_{R}^{i}$ starting with the instance with the highest nonconformity score until the $p$-value of $S_{R}^{i}$ equals or exceeds $\epsilon_{t}$. We note that this process is sequential due to the fact that $t\left(T^{i}, S_{s}^{i}\right)$ is not monotonic for the nonconformity scores smaller than the largest target nonconformity score $\alpha_{T_{\max }}^{i}$. Finally, the class relevance of $X^{i}$ is estimated on $T^{i} \cup S_{R}^{i}$ using the class relevance measure of choice.

\section{Experiments}

This section presents our experimental set-up, results and conclusions. We first introduce the instance-transfer tasks under study in Section 6.1. The experimental set-up is provided in Section 6.2. In Section 6.3, we analyze the generalization performance of the proposed instance-transfer decision trees. Section 6.4 discusses the influence of threshold $\lambda$ that controls the minimum number of target instances per class needed to allow instance transfer.

\subsection{Instance-transfer classification tasks}

In our experiments, we considered six instance-transfer classification tasks defined on realworld data sets that are commonly used in transfer learning research. Each task was given with a target set and a source set, described in Table 1. The instance-transfer tasks are defined below.

- The first instance-transfer classification task was the landmine detection task [1]. It is a task of detecting landmine in different landmine fields. There are 29 data sets sampled from 29 landmine fields. The 29 sets have different distributions due to various ground surface conditions. For example, the data sets "Mine 1" to "Mine 15" correspond to foliated regions, while the data sets "Mine 16" to "Mine 29" correspond to regions that have bare earth. In our experiment, the data set "Mine 29" was used as the target data and the data set "Mine 1" was used as the source data. To guarantee that the target data and the source data are distributed differently for some features, we manipulated the marginal distribution of the feature with the highest gain ratio of the source data by adding random noise generated from the standard uniform distribution.

- The second instance-transfer classification task was to estimate correctness of the diagnosis for a patient treated by a medical doctor [24]. The data consists of records of patients treated by two different doctors. Each patient record was regarded as an instance that is represented by 18 bio-markers and a class label indicating whether the corresponding doctor had provided the correct diagnosis. The instances were grouped 


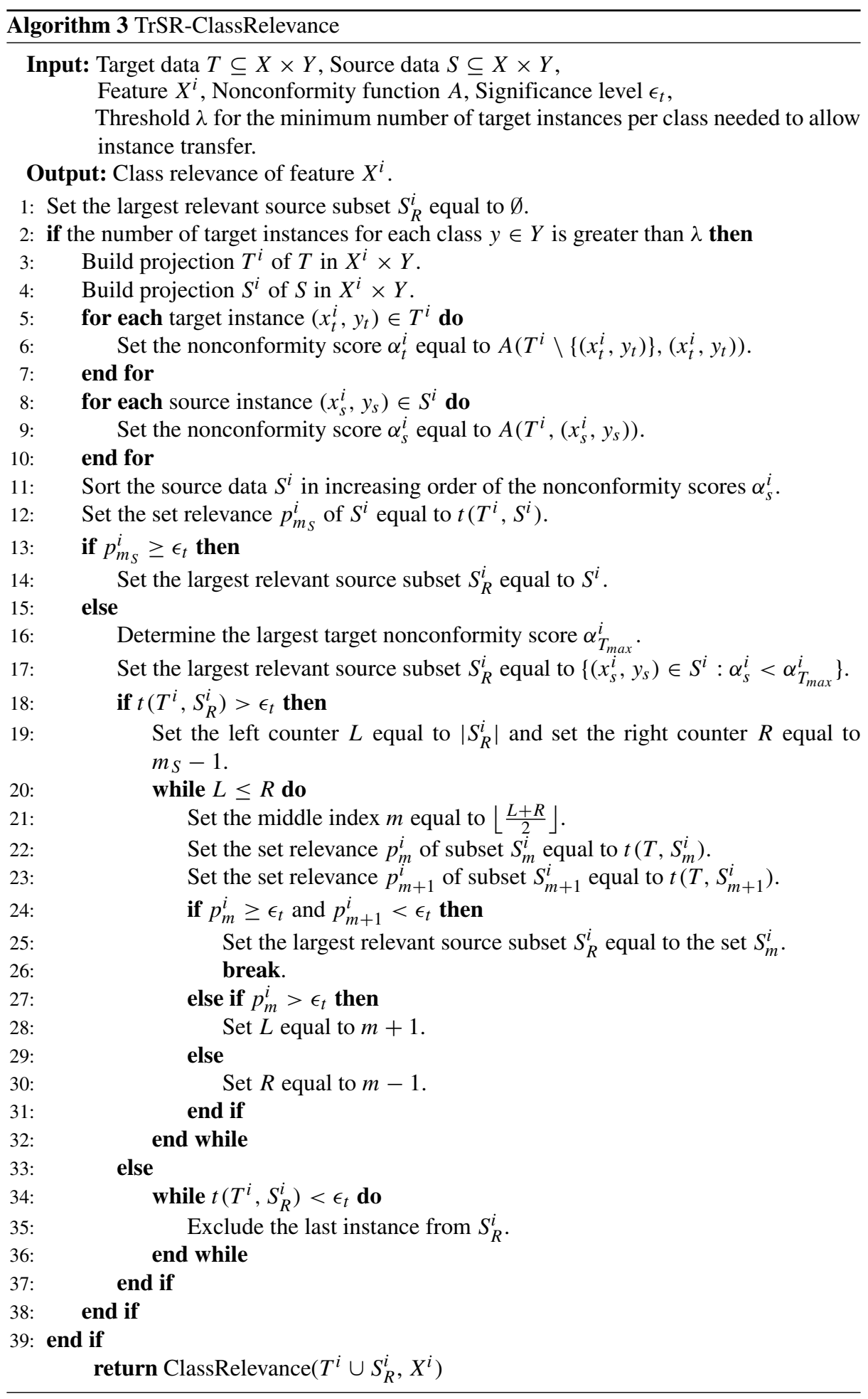


Table 1 Descriptions of the data sets for instance-transfer classification tasks

\begin{tabular}{llll}
\hline Task & $\begin{array}{l}\text { Number of } \\
\text { classes }\end{array}$ & $\begin{array}{l}\text { Data set size } \\
|T|\end{array}$ & $|S|$ \\
\hline Landmine & 2 & 449 & 690 \\
Medical Center & 2 & 81 & 453 \\
Wine Quality & 3 & 159 & 1499 \\
USPS & 10 & 183 & 1820 \\
Student 1 & 2 & 46 & 46 \\
Student 2 & 2 & 46 & 349 \\
\hline
\end{tabular}

per doctor. We used the group with comparably small size as the target set and the other one as the source set.

- The third instance-transfer classification task was the handwritten digital recognition task from the USPS corpus [11]. It is a task of recognizing handwritten digits (0 to 9) automatically scanned from envelopes by the U.S. Postal Service. We applied principal component analysis $(P C A)$ to project the original $16 \times 16$ gray scale images to a feature space of 10 dimensions. The digit IDs (0-9) were considered as classes. A target set and a source set were randomly drawn from the transformed USPS corpus. Random noise generated from the standard uniform distribution was added to two features corresponding to highest eigenvalues of the source data to guarantee that the target and source data were related w.r.t a strict subset of features.

- The fourth instance-transfer classification task was wine quality classification [5]. This task is based on a collection of instances related to red and white variants of Portuguese wine. Each instance is represented by 11 physiochemical features (e.g. PH values) and a grade given by experts between 0 (very bad) and 10 (very excellent). In our experiments, we defined a multi-class classification task based on the grades, i.e. labeling the instances with grade 0 to 4, 5 to 6, and 7 to 10 as "poor", "normal", and "excellent", respectively. A random sample from the red wine data was used as the target data and a random sample of the white wine data was used as the source data. To better fit the scenario that the source data are partially input-feature relevant to the target data, random noise generated from the standard uniform distribution was added to the two features with the highest gain ratios of the source data.

- The fifth and sixth instance-transfer classification tasks were defined on the exam records of students from two Portuguese schools: Gabriel Pereira and Mousinho da Silveira [6]. Each exam record was considered as an instance that is represented by a series of demographic, social, and school related features and a binary grade (pass or no pass). In our experiments, we defined a binary classification task on the grades. The two instance-transfer tasks were defined as follows: the fifth task (referred to as Student 1) used the students' Mathematics exam records of school Mousinho da Silveira as the target data, and used the Portuguese exam records of the same group of students as the source data; the sixth task (referred to as Student 2) employed the same target data as the first task, but used the students' Mathematics exam records of school Gabriel Pereira as the source data.

\subsection{Experimental set-up}

For all six classification tasks, we implemented the four types of instance-transfer decision trees described in Section 5.2: 
- PreIR-DT: decision trees with pre-training source-subset selection based on individual relevance;

- PreSR-DT: decision trees with pre-training source-subset selection based on set relevance;

- EmbedIR-DT: decision trees with embedded source-subset selection based on individual relevance;

- EmbedSR-DT: decision trees with embedded source-subset selection based on set relevance.

All decision trees were induced using the well-known decision tree algorithm C4.5 [16] (described in Section 5.1). The class relevance of features was evaluated in terms of Gain Ratio, which is featured in C4.5. The threshold $\lambda$ in EmbedIR-DT and EmbedSR-DT was set to 10 .

In the experiments, we approximated the $p$-value function by the Wilcoxon rank-sum test (described in Section 4.2). To approximate the $p$-value we first needed to set up the instance nonconformity function. This set-up was done depending on the type of feature. For numerical features, the nearest-neighbor instance nonconformity function [18] was employed. For a target data set $T$ and an instance $\left(x_{s}, y_{s}\right)$, the nearest neighbor instance nonconformity function $A_{N N}$ outputs a nonconformity score $\frac{\sum_{j=1}^{k} d_{s j}^{+}}{\sum_{j=1}^{k} d_{s j}^{-}}$, where $k$ is the number of nearest neighbors, $d_{s j}^{+}$is the $j$-th shortest distance from $x_{s}$ to some instances in $T$ having the same class label as $x_{s}$, and $d_{s j}^{-}$is the $j$-th shortest distance from $x_{s}$ to some instances in $T$ having different class labels. For nominal features, the nonconformity score of an instance $\left(x_{s}, y_{s}\right)$ is computed as: $\frac{\sum_{\bar{y} \in Y \backslash\left\{y_{s}\right\}} P\left(\bar{y} \mid x^{i}\right)}{P\left(y \mid x^{i}\right)}$, where $P\left(y \mid x^{i}\right)$ is the normalized count of class $y$ given the feature $x^{i}$.

The method of evaluation was 10 -fold cross validation on the target data repeated 50 times; i.e., the source data were used as auxiliary training data. The generalization performance of the instance-transfer classifiers was evaluated using Area Under the ROC Curve (AUC). The performance of $\mathrm{C} 4.5$ decision trees for the case of no instance transfer was used as baseline. A paired t-test was performed with significance level 0.05 to find significantly better (or worse) results w.r.t the baseline classifier.

The instance-transfer decision trees were also compared with the six instance-transfer approaches presented in Section 2. Two of them are feature selection based approaches: MMDE and f-MMD. We set the dimension of the reduced feature space to 10 for MMDE. ${ }^{3}$ In $\mathrm{f}-\mathrm{MMD}$, we excluded the features which weights are higher than 0.1 . Decision trees were used to build prediction models in a low-dimensional space. The other four of them are source-subset selection based approaches: TrAdaBoost, Dynamic-TrAdaBoost, TraBagg and DoubleBootStrap. All the four approaches used decision trees as base classifiers. The number of iterations was set to 100 for all of them.

\subsection{Experimental results}

We experimentally compared the performance of instance-transfer decision trees PreIR-DT, PreSR-DT, EmbedIR-DT and EmbedSR-DT. The AUCs of all those classifiers are given at significance level $\epsilon_{t}=0.5$ for source-subset selection. The reason is twofold. Firstly, if a source instance (subset) is generated by the target distribution, the expected $p$-value

\footnotetext{
${ }^{3}$ The dimension of the reduced feature space was set to 8 for Landmine and USPS tasks, since their original dimension is less or equal to 10 .
} 
of this instance (subset) is equal to 0.5. Secondly, as mentioned in Section 5 set relevance is computationally expensive due to the non-monotonicity of the set $p$-values. However, selecting a subset with set relevance equal to 0.5 is relatively simple.

The results for our decision-tree approaches and for the other six instance-transfer approaches described in Section 2 are given in Table 2. From the table we see that EmbedSR-DT achieves the best generalization performance over all the instance-transfer classification tasks. It is significantly better than the baseline (for the case of no instance transfer) for all tasks. It gains a margin of 0.08 over the AUC of the baseline classifier in the best case (the medical center task). EmbedIR-DT has the second best generalization performance (significantly better than the baseline classifier in 5 out of 6 tasks).

Comparing with the feature selection based approaches (i.e., MMDE and f-MMD), source-subset selection based approaches provide more promising results. That is because the benefit the feature selection based approaches gain from source instances cannot always compensate the information loss that they suffer from dimensional reduction.

Comparing our approaches with other source-subset selection based approaches, we find that EmbedSR-DT and EmbedIR-DT clearly outperform all other approaches and never result in negative transfer. Especially for the Student 1 task, EmbedSR-DT and EmbedIRDT give significant improvement while other approaches give no improvement or even result in negative instance transfer (e.g. TrAdaBoost, Dynamic-TrAdaBoost and TraBagg). This observation confirms the main claim of this paper that our instance-transfer decision trees that employ embedded selection are more promising when the target and source data are relevant for strict subsets of input features.

We further compared the performance of our instance-transfer decision trees PreIR-DT, PreSR-DT, EmbedIR-DT and EmbedSR-DT on different significance levels $\epsilon_{t}$ for source selection. Figure 1a presents the performance of these four classifiers employing $\epsilon_{t}$ ranging from 0 to 1 with step 0.1 for the medical center task. As we can see from Fig. 1a, decision trees with embedded source-subset selection achieve better AUCs than those with pretraining selection most of the time which reinforces the main claim of this paper. Moreover, we note that decision trees that employ set relevance for source-subset selection outperform those employing individual relevance (i.e., EmbedSR-DT outperforms EmbedIR-DT, and PreSR-DT outperforms PreIR-DT) when the significance level $\epsilon_{t}$ is relatively high, e.g, $\epsilon_{t} \geq 0.5$. This is because the approaches that employ individual relevance are more conservative: they select a subset of the source instances selected by the approaches that employ set relevance. To illustrate this fact, we give an example for the medical center task in Fig. 2. We sorted the instances from the source data in increasing order of the nonconformity scores. Then, we added the source instance with the lowest nonconformity score to a preliminary empty source subset and computed the set $p$-value. We repeated the last step until all the source instances were added, and we plotted the obtained set $p$-values against the size of the source subsets in Fig. 1b. After that, we repeated the same process for individual relevance, i.e., instead of computing set $p$-values we computed the individual $p$-value for the last instance of each subset and plotted it in Fig. 1b. Comparing the plots of set $p$-values and individual $p$-values, we observe that the set $p$-value is much bigger than the individual $p$-value for the same subset. Thus, when $\epsilon_{t} \geq 0.5$ PreIR-DT and EmbedIR-DT select less instances from the sorted source which implies that they benefit less from the relevant source data. ${ }^{4}$

\footnotetext{
${ }^{4}$ When the significance level is set to 0.5 or a higher value, the source instances are more likely to be generated from the target distribution, and thus we say they are relevant source instances.
} 


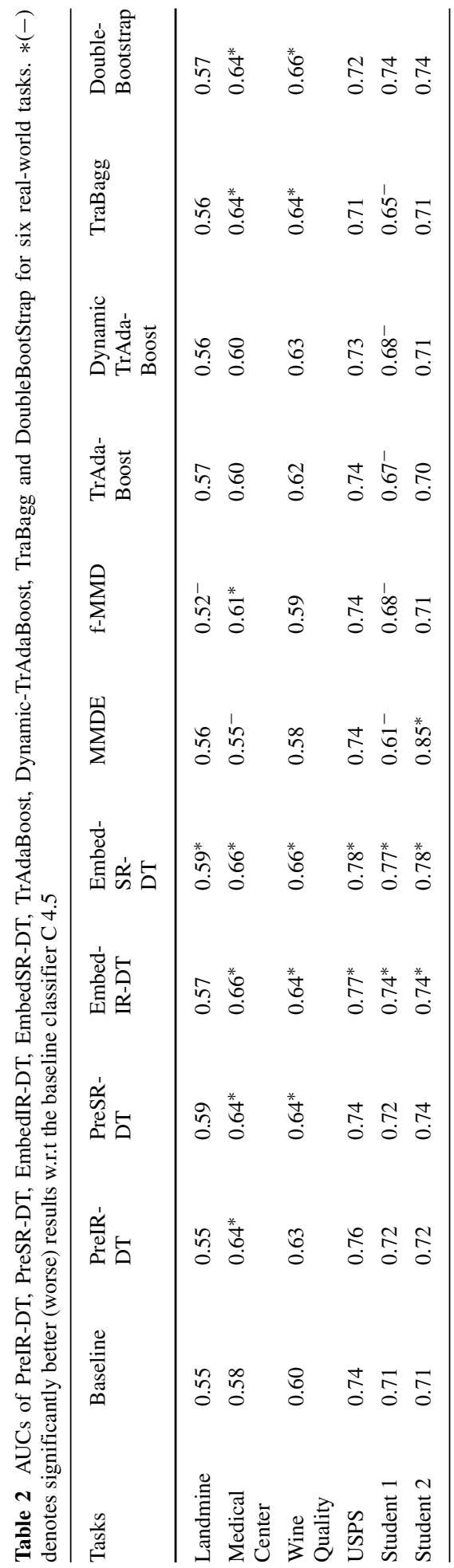




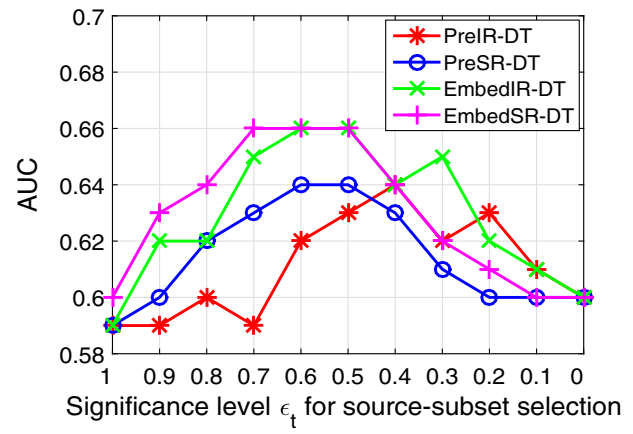

(a)

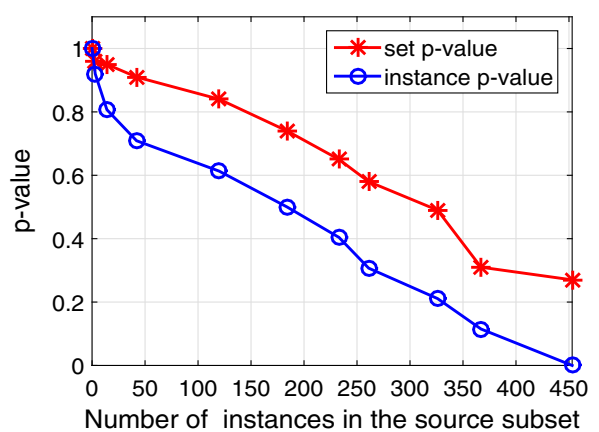

(b)

Fig. 1 (a): AUCs of instance-transfer decision trees employing decreasing $\epsilon_{t}$ for the medical center task. (b): Individual $p$-value and set $p$-value plotted against the size of the selected source subset

\subsection{Influence of minimum size of target data for transfer}

The size of the target data plays an important role for instance transfer. This is due to the fact that if this size is small, estimated $p$-values can be imprecise. To avoid this negative effect, we introduced a threshold $\lambda$ for the minimal number of target instances needed to allow instance transfer. In Fig. 2a and b, we study the influence of $\lambda$ on instance transfer. More precisely, the figures present the average AUCs of EmbedSR-DT and EmbedIR-DT for $\lambda$ ranging from 10 to 50 with step 5 for the wine quality classification and the medical center task, respectively. The significance level $\epsilon_{t}$ for instance transfer is set to 0.5 .

In Fig. 2a, the plots show that the performance of EmbedSR-DT and EmbedIR-DT improves as the value of $\lambda$ increases from 5 to 25 . That is because the estimations of relevance of source instances become more accurate as the size of target training data gets bigger. However, the AUCs drop when $\lambda$ exceeds 25 . The reason is that instance transfer is only allowed at high-level nodes of the tree when $\lambda$ is bigger than 25 . Therefore, the final model benefits less from instance transfer. When $\lambda$ increases to 45 or more, there is no instance transfer at all. A similar pattern can be observed in Fig. 2b. EmbedSR-DT and EmbedIR-DT achieve the best generalization performance when $\lambda$ equals 10 . Then, the AUCs drop as the value of $\lambda$ increases. When $\lambda$ goes above 35 , there are not enough target

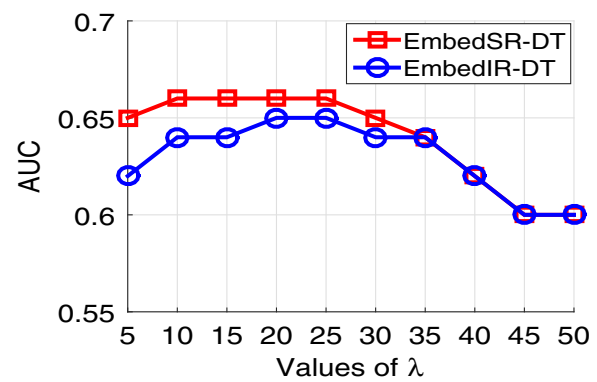

(a)

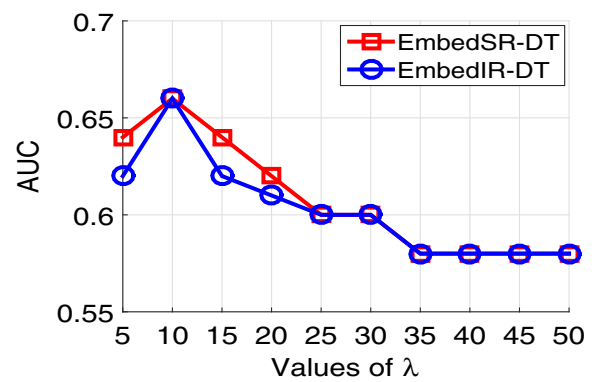

(b)

Fig. 2 (a): AUCs of EmbedSR-DT and EmbedIR-DT for different values of $\lambda$ for the wine quality classification. (b): AUCs of EmbedSR-DT and EmbedIR-DT for different values of $\lambda$ for the medical center task 
instances per class thus instance transfer is not performed. Based on these observations, we conclude that to achieve the best instance-transfer result, the threshold $\lambda$ needs to be well tuned to control the trade-off between the accuracy of relevance estimation and the amount of source instances being transferred.

\section{Conclusion}

This paper proposed a decision-tree approach to overcome the problem of sub-optimal instance transfer and the problem of negative instance transfer when the source data are partially input-feature relevant to the target data. The approach embeds univariate instance transfer for feature selection at tree nodes. To provide a statistical guarantee for the relevance to the target data, the instance transfer is guided by a conformal test. The test estimates the relevance of source instances either on the individual level, or on the set level given at least $\lambda$ target instances. It was shown that the estimation is more accurate on the set level when this user-selected threshold $\lambda$ is properly tuned (i.e., when it provides a good trade-off between the error of relevance estimation and the number of source instances to be transferred). The experiments showed that instance transfer approaches that are based on source-subset selection outperform those based on feature selection. Our decision trees with embedded source-subset selection achieve the best results compared with other source-subset selection based approaches. The advantage of our decision trees over other approaches is more obvious for tasks that are characterized by partial input-feature relevance of the target and source data.

Future research will follow two main directions. The first one is to reduce the computational complexity of the source-subset selection based on set-relevance by designing efficient approximations to find the largest relevant source subsets. The second direction is to develop new instance-transfer approaches for partially input-feature relevant source and target data. For example, univariate instance transfer can be applied to other standard machine learning models, such as: NaiveBayes, KNN, etc.

Open Access This article is distributed under the terms of the Creative Commons Attribution 4.0 International License (http://creativecommons.org/licenses/by/4.0/), which permits unrestricted use, distribution, and reproduction in any medium, provided you give appropriate credit to the original author(s) and the source, provide a link to the Creative Commons license, and indicate if changes were made.

\section{References}

1. Landmine detection data. http://www.ee.duke.edu/lcarin/landminedata.zip

2. Al-Stouhi, S., Reddy, C.K.: Adaptive boosting for transfer learning using dynamic updates. In: Machine Learning and Knowledge Discovery in Databases, pp. 60-75. Springer (2011)

3. Aldous, D.J.: Exchangeability and related topics. In: École D'été De ProbabilitéS De Saint-Flour, XIII1983, vol. 1117, pp. 1-198. Springer, Berlin (1985)

4. Borgwardt, K.M., Gretton, A., Rasch, M.J., Kriegel, H.P., Schölkopf, B., Smola, A.J.: Integrating structured biological data by kernel maximum mean discrepancy. Bioinformatics 22(14), e49-e57 (2006)

5. Cortez, P., Cerdeira, A., Almeida, F., Matos, T., Reis, J.: Modeling wine preferences by data mining from physicochemical properties. Decis. Support. Syst. 47(4), 547-553 (2009)

6. Cortez, P., Silva, A.M.G.: Using data mining to predict secondary school student performance. EUROSIS (2008)

7. Dai, W., Yang, Q., Xue, G.R., Yu, Y.: Boosting for transfer learning. In: Proceedings of the 24th International Conference on Machine Learning, pp. 193-200. ACM (2007) 
8. Jolliffe, I.: Principal component analysis. Wiley Online Library (2002)

9. Kamishima, T., Hamasaki, M., Akaho, S.: Trbagg: a Simple Transfer Learning Method and Application to Personalization in Collaborative Tagging. In: Proceedings of the 9th IEEE International Conference on Data Mining, pp. 219-228 (2009)

10. Lehmann, E.L., D’Abrera, H.J.: Nonparametrics: Statistical Methods based on Ranks. Springer, New York (2006)

11. Lichman, M.: UCI Machine learning repository. http://archive.ics.uci.edu/ml (2013)

12. Lin, D., An, X., Zhang, J.: Double-bootstrapping source data selection for instance-based transfer learning. Pattern Recogn. Lett. 34(11), 1279-1285 (2013)

13. Pan, S.J., Kwok, J.T., Yang, Q.: Transfer learning via dimensionality reduction. In: AAAI, vol. 8, pp. 677-682 (2008)

14. Pan, S.J., Tsang, I.W., Kwok, J.T., Yang, Q.: Domain adaptation via transfer component analysis. IEEE Trans. Neural Netw. 22(2), 199-210 (2011)

15. Pan, S.J., Yang, Q.: A survey on transfer learning. IEEE Trans. Knowl. Data Eng. 22(10), 1345-1359 (2010)

16. Quinlan, J.R.: C4.5: Programs for machine learning. Morgan Kaufmann Publishers Inc, San Mateo (1993)

17. Schapire, R.E.: The strength of weak learnability. Mach. Learn. 5(2), 197-227 (1990)

18. Shafer, G., Vovk, V.: A tutorial on conformal prediction. J. Mach. Learn. Res. 9, 371-421 (2008)

19. Torrey, L., Shavlik, J.: Transfer learning. Handbook of Research on Machine Learning Applications and Trends: Algorithms, Methods, and Techniques 1, 242 (2009)

20. Uguroglu, S., Carbonell, J.: Feature selection for transfer learning. In: Joint European Conference on Machine Learning and Knowledge Discovery in Databases, pp. 430-442. Springer (2011)

21. Vovk, V.: The basic conformal prediction framework. In: Conformal Prediction for Reliable Machine Learning Theory, Adaptations and Applications, pp. 1-20 (2014)

22. Zhou, S., Smirnov, E., Schoenmakers, G., Driessens, K., Peeters, R.: Testing exchangeability for transfer decision. Pattern Recognit. Lett. 88(C), 64-71 (2017)

23. Zhou, S., Smirnov, E., Schoenmakers, G., Peeters, R.: Conformity-based source subset selection for instance transfer. Neurocomputing. doi:10.1016/j.neucom.2016.11.071 (2017)

24. Zhou, S., Smirnov, E.N., Peeters, R.: Conformal region classification with instance-transfer boosting. Int. J. Artif. Intell. Tools 24(6), 1560,002 (2015) 